\title{
RCSEd welcomes Scottish Government anti-bullying group
}

In response to a review led by John Sturrock QC into allegations of bullying in NHS Highland, an advisory group has been established by the Scottish Government to promote professional behaviours and improve working practices.

The Royal College of Surgeons of Edinburgh (RCSEd), which has been tackling these issues with its \#LetsRemoveIt campaign, welcomes the Scottish Government's initiative in taking the Sturrock Report forward to resolve bullying and undermining across NHS Scotland. Results of the group include appointing whistleblowing champions to each health board and bringing together a wide range of organisations to work collaboratively on these important issues.

RCSEd has been addressing bullying and undermining with its \#LetsRemoveIt campaign since 2017 after its membership survey reported that $40 \%$ of respondents had been victims of such behaviour and the same amount had witnessed bullying and undermining.

The campaign was nominated for British Medical Journal (BMJ) and Health Service Journal (HSJ) awards in recognition of the excellent resources, events and materials it offers healthcare professionals and

\section{BOOK REVIEW}

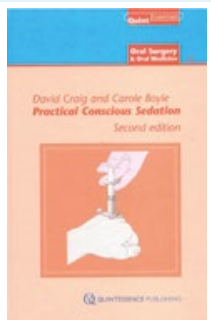

\section{PRACTICAL CONSCIOUS SEDATION, SECOND EDITION}

David Craig, Carole Boyle;

2017; Quintessence Publishing;

price $\mathrm{f} 28.00 \mathrm{pp} .134$;

ISBN: 9781786980052

Practical conscious sedation proves a concise yet comprehensive tool for dentists and dental students alike wishing to review and develop their knowledge of conscious sedation. The book provides a broad outline of the history of conscious sedation in dentistry and discusses anatomy, physiology and pharmacology relevant to the provision of conscious sedation in general dental practice. Thereafter, the practical techniques and requirements for training are described, including the management of associated complications.

The book begins by outlining the historical development of conscious sedation techniques in dentistry with emphasis on those still in use today such as intravenous sedation (IVS) with midazolam and nitrous oxide inhalation sedation (IHS). The ensuing chapters review basic physiology and anatomy pertinent to the practice of sedation. This serves to revise the respiratory and cardiovascular physiology studied at dental school while describing in more depth the anatomy of superficial veins in the forearm and hands to assist with successful cannulation.

The subsequent chapter revises pharmacology previously outlined in the undergraduate curriculum with specific emphasis on benzodiazepines and nitrous oxide and their mechanism of action. Other sedation techniques that can be employed by anaesthetic teams, such as propofol and midazolam combined with opioids, are discussed and the indications for such provision outlined. In addition, a potential future IHS alternative to nitrous oxide, sevoflurane in oxygen is described.

A chapter is devoted to initial assessment and treatment planning. This chapter outlines the important factors to consider when triaging
\#LetsRemovelt

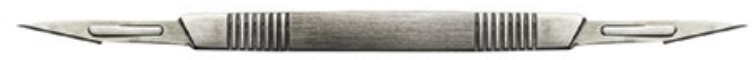

Bullying harms your profession and your patients.

Trusts, including advice for bullies to improve their behaviours. The advisory group includes a wide range of senior stakeholders. Professor Michael Griffin OBE, President of RCSEd and \#LetsRemoveIt campaigner, said: 'I'm happy to see the Scottish Government are taking bullying and undermining within the NHS seriously. This is a positive step forwards and will bring organisations together to collaborate on future anti-bullying and undermining work. The \#LetsRemoveIt campaign has highlighted the need for action and we will continue leading the way in this area.' For more information about the \#LetsRemoveIt campaign visit https:// www.rcsed.ac.uk/professional-support-development-resources/ anti-bullying-and-undermining-campaign.

patients for conscious sedation in general dental practice. The importance of comprehensive history taking is noted. The medical considerations and ASA classification that must be utilised as part of the pre-operative assessment for dental treatment are provided including the need for baseline observations to be recorded. Social history taking helps to determine whether support and suitable escorts are available in the post sedation period.

Subsequent chapters revise the armamentarium required for IHS and IVS provision previously studied at dental school. The mandatory requirements for pulse oximetry to be undertaken during IVS and for such practitioners to have intermediate life support training are duly noted. Colourful images augment the reader's understanding throughout.

There are dedicated chapters outlining the clinical techniques of IHS and IVS, including pre-operative checklists, venepuncture, monitoring, recovery and discharge. Furthermore, specific guidance is provided for the indications of midazolam reversal with flumazenil. Techniques not routinely employed by operator-sedationist trained dentists such as oral and intranasal midazolam are also described.

The final chapters detail complications of conscious sedation. These range from the management of respiratory depression and airway obstruction to venepuncture difficulties. Subsequent chapters discuss patient co-morbidities that necessitate referral to secondary care and the indications for general anaesthesia as opposed to conscious sedation. The final chapter outlines contemporaneous standards of good practice and medicolegal considerations. Current guidelines that dentists working in the UK are expected to follow are described for sedation in dentistry. This is an excellent resource for dentists that have qualified outside the UK.

This book provides an excellent foundation in conscious sedation techniques, consolidating and expanding upon the undergraduate dental school curriculum. The information provided also serves as an invaluable update and revision tool for general dental practitioners and dentists in training posts with limited exposure to conscious sedation techniques.

Claire King 